\title{
Observations of MeV electrons in Jupiter's innermost radiation belts and polar regions by the Juno radiation monitoring investigation: Perijoves 1 and 3
}

Becker, Heidi N.; Santos-Costa, Daniel; Jørgensen, John Leif; Denver, Troelz; Adriani, Alberto; Mura, Alessandro; Connerney, John E. P.; Bolton, Scott J.; Levin, Steven M.; Thorne, Richard M.

Total number of authors:

19

Published in:

Geophysical Research Letters

Link to article, DOI:

10.1002/2017GL073091

Publication date:

2017

Document Version

Publisher's PDF, also known as Version of record

Link back to DTU Orbit

Citation $(A P A)$ :

Becker, H. N., Santos-Costa, D., Jørgensen, J. L., Denver, T., Adriani, A., Mura, A., Connerney, J. E. P., Bolton, S. J., Levin, S. M., Thorne, R. M., Alexander, J. W., Adumitroaie, V., Manor-Chapman, E. A., Daubar, I. J., Lee, C., Benn, M., Sushkova, J., Cicchetti, A., \& Noschese, R. (2017). Observations of MeV electrons in Jupiter's innermost radiation belts and polar regions by the Juno radiation monitoring investigation: Perijoves 1 and 3 . Geophysical Research Letters, 44(10), 4481-4488. https://doi.org/10.1002/2017GL073091

\section{General rights}

Copyright and moral rights for the publications made accessible in the public portal are retained by the authors and/or other copyright owners and it is a condition of accessing publications that users recognise and abide by the legal requirements associated with these rights.

- Users may download and print one copy of any publication from the public portal for the purpose of private study or research.

- You may not further distribute the material or use it for any profit-making activity or commercial gain

- You may freely distribute the URL identifying the publication in the public portal 


\section{Geophysical Research Letters}

\section{RESEARCH LETTER}

10.1002/2017GL073091

Special Section:

Early Results: Juno at Jupiter

Key Points:

- High-latitude sampling of relativistic electrons at the inner edge of the Jovian radiation belts is discussed

- Noise from penetrating radiation is used to detect $\mathrm{MeV}$ electrons

- Enhanced particle counts were observed in Jupiter's polar regions

Supporting Information:

- Supporting Information S1

Correspondence to:

H. N. Becker,

heidi.n.becker@jpl.nasa.gov

\section{Citation:}

Becker, H. N. et al (2017), Observations of MeV electrons in Jupiter's innermost radiation belts and polar regions by the Juno radiation monitoring investigation: Perijoves 1 and 3, Geophys. Res. Lett., 44, 4481-4488, doi:10.1002/ 2017GL073091.

Received 14 FEB 2017 Accepted 12 APR 2017 Published online 25 MAY 2017

(c)2017. American Geophysical Union. All Rights Reserved.

\section{Observations of MeV electrons in Jupiter's innermost radiation belts and polar regions by the Juno radiation monitoring investigation:} Perijoves 1 and 3

\author{
Heidi N. Becker ${ }^{1}$ (D), Daniel Santos-Costa ${ }^{2}$ D, John L. Jørgensen ${ }^{3}$, Troelz Denver ${ }^{3}$ (D), \\ Alberto Adriani $^{4}$ (D), Alessandro Mura ${ }^{4}$ (D) John E. P. Connerney ${ }^{5,6}$ (D), Scott J. Bolton ${ }^{2}$ (D, \\ Steven M. Levin ${ }^{1}$ D, Richard M. Thorne ${ }^{7}$ (D, James W. Alexander ${ }^{1}$, Virgil Adumitroaie ${ }^{1}$ (D), \\ Emily A. Manor-Chapman ${ }^{1}$ (D), Ingrid J. Daubar ${ }^{1}$ (D, Clifford Lee', Mathias Benn ${ }^{3}$ (D), Julia Sushkova ${ }^{3}$, \\ Andrea Cicchetti ${ }^{4}$ iD, and Raffaella Noschese ${ }^{4}$
}

\begin{abstract}
${ }^{1}$ Jet Propulsion Laboratory, California Institute of Technology, Pasadena, California, USA, ${ }^{2}$ Southwest Research Institute, San Antonio, Texas, USA, ${ }^{3}$ DTU Space, National Space Institute, Technical University of Denmark, Kgs. Lyngby, Denmark, ${ }^{4}$ INAF, Istituto di Astrofisica e Planetologia Spaziali, Rome, Italy, ${ }^{5}$ Space Research Corporation, Annapolis, Maryland, USA, ${ }^{6}$ NASA Goddard Space Flight Center, Greenbelt, Maryland, USA, ${ }^{7}$ Department of Atmospheric and Oceanic Sciences, University of California, Los Angeles, California, USA
\end{abstract}

\begin{abstract}
Juno's "Perijove 1" (27 August 2016) and "Perijove 3" (11 December 2016) flybys through the innermost region of Jupiter's magnetosphere (radial distances $<2$ Jovian radii, $1.06 R_{J}$ at closest approach) provided the first in situ look at this region's radiation environment. Juno's Radiation Monitoring Investigation collected particle counts and noise signatures from penetrating high-energy particle impacts in images acquired by the Stellar Reference Unit and Advanced Stellar Compass star trackers, and the Jupiter Infrared Auroral Mapper infrared imager. This coordinated observation campaign sampled radiation at the inner edges of the high-latitude lobes of the synchrotron emission region and more distant environments. Inferred omnidirectional $>5 \mathrm{MeV}$ and $>10 \mathrm{MeV}$ electron fluxes derived from these measurements provide valuable constraints for models of relativistic electron environments in the inner radiation belts. Several intense bursts of high-energy particle counts were also observed by the Advanced Stellar Compass in polar regions outside the radiation belts.
\end{abstract}

\section{Introduction}

Jupiter's inner magnetosphere ( $<5 R_{\mathrm{J}}$, radial distance from Jupiter's center) is largely unexplored. Our understanding of the relativistic electron population within the inner radiation belts has been guided by in situ measurements obtained during three prior missions and remote observations of synchrotron emission (Bolton et al. [2004] provide a general review). Inside $\sim 3.5 R_{\mathrm{J}}$ " the synchrotron region," synchrotron emission from relativistic electrons contributes to the Jovian radio emission [Carr et al., 1983]. Earth-based observations of synchrotron emission have been used since the 1950s to study the spatial and energy distributions of high-energy electrons in the inner radiation belts. A structure of intense emission near the magnetic equator $\left(\sim 1.5 R_{\jmath}\right)$ as well as high-latitude lobes has been observed. Synchrotron emission provides constraints for models of pitch angle distributions and electron energy spectra and gives hints about the physical mechanisms governing the distributions. However, interferometric image resolution is typically $\geq \sim 0.25 R$, and removing Jovian thermal emission from the higher-frequency data (from electrons greater than several tens of $\mathrm{MeV}$ ) is challenging. Therefore, the finer structure remains unclear and in situ measurements provide important checks.

In 1973 Pioneer 10 sampled energetic particles during a low inclination flyby inward to $2.85 R$. Pioneer 11 measured energetic particles during its high inclination flyby, passing to within $\sim 1.6 R$, of Jupiter in 1974 $\left(-13^{\circ}\right.$ latitude at closest approach), and the Galileo probe sampled energetic particles along its approximately equatorial trajectory into Jupiter's atmosphere in 1995 [Bolton et al., 2004; Fischer et al., 1996] (data from the two Galileo orbiter passes inside $2.5 R$, have been difficult to use [Garrett et al., 2012]). However, these three encounters provide limited coverage of this complex environment. No nonequatorial in situ data have previously been collected inside $1.6 R$ J. The inner edge of Jupiter's radiation belts is almost entirely unsampled. Juno's unprecedented polar orbit enables such data collection at high magnetic latitudes, 


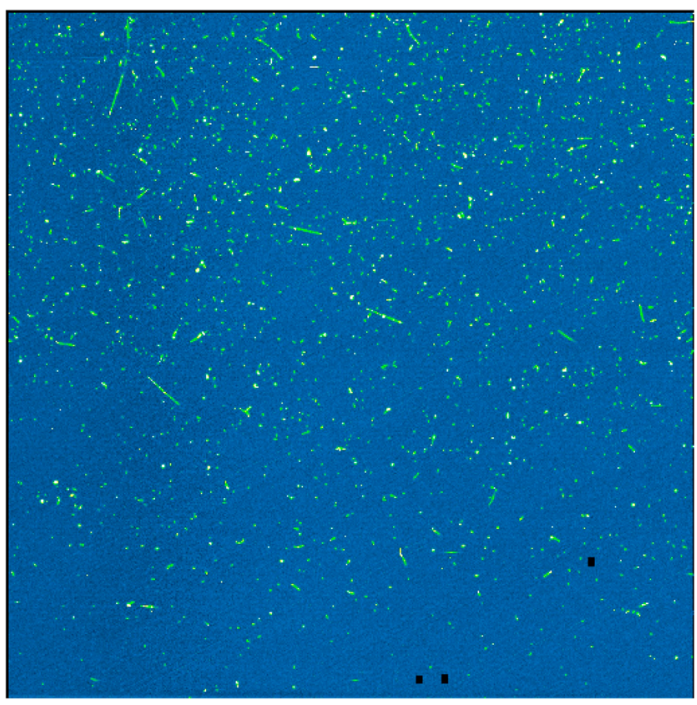

Figure 1. SRU CCD image collected at 13:18:16.336 UTC 27 August 2016 within Jupiter's inner radiation belts $\left(1.4 R j ;-54.3^{\circ}\right.$ dipole magnetic latitude, derived from VIP4 [Connerney et al., 1998]). The image illustrates characteristic imager noise signatures due to penetrating relativistic electrons (bright dots, streaks, and squiggles). Three small pixel regions in the lower part of the image (black squares) were removed prior to particle count rate processing due to the presence of star signal above the particle noise detection threshold. bles in collaborative observation campaigns, collecting "radiation images" and penetrating particle counts at targeted locations within the magnetosphere during each of Juno's perijove passes. Instruments contributing to the campaigns include the spacecraft Stellar Reference Unit (SRU), the Magnetic Field Investigation's Advanced Stellar Compass (ASC) camera head D imager [Connerney et al., 2017], and the Jupiter Infrared Auroral Mapper (JIRAM) infrared imager [Adriani et al., 2014]. A combination of onboard and ground-based image processing is used to extract the characteristic signatures of penetrating high-energy electrons (Figure 1) and derive count rates to characterize the external environment [Becker et al., 2017]. In this letter we discuss the first high-latitude sampling of $>10 \mathrm{MeV}$ electrons at the innermost edge of the Jovian radiation belts and observations of high-energy penetrators in Jupiter's polar regions during Juno's Perijove 1 and Perijove 3 flybys.

\section{Methodology}

Geant4 shielding analysis [Allison et al., 2006] was performed for each instrument to characterize the penetration efficiencies of omnidirectional high-energy electrons and protons as a function of their external energy (before entering the instrument). These probabilities were interpolated over energy and integrated using modeled Jovian environment spectra from Garrett et al. [2005], Divine and Garrett [1983], and Garrett et al. [2003] to assess instrument count rate response in similar environments. We also assessed smaller energy regimes within modeled spectra to see how each external energy "bin" contributed to the total count (accounting for contributions by primaries and secondaries). This revealed the higher-energy regimes which make the dominant contributions to the overall count, allowing us to identify an approximate energy channel and count rate metric for each instrument. The metrics allow the external electron flux to be inferred from the count rates of noise events observed in the respective images (Table 1). We assume that penetrating ions contribute negligible counts given their expected intensities under the shielding.

The spectra from the cited models are not without uncertainty as they are based on limited samples from integral channels no higher than $>31 \mathrm{MeV}$, and pitch angle distributions were adjusted to qualitatively match ground observed synchrotron emission. However, an initial assumption using plausible models was necessary to establish a baseline calibration to seed the interpretation of our data. While RM count rates are 
Table 1. Approximate Energy Channels and Measurement Ranges of Juno RM Instruments [Becker et al., 2017] ${ }^{\mathrm{a}}$

\begin{tabular}{lccc} 
Instrument & $\begin{array}{c}\text { Energy Sensitivity } \\
\text { (Electrons) }\end{array}$ & $\begin{array}{c}\text { Measurement Range } \\
\text { (Count Rate) }\end{array}$ & $\begin{array}{c}\text { Inferred External Omnidirectional Flux } \\
\left(\mathrm{el}^{-2} \mathrm{sm}^{-1} \text { ) }\right.\end{array}$ \\
\hline SRU CCD imager & $>10 \mathrm{MeV}$ & $4 \mathrm{E} 2-1.6 \mathrm{E} 7$ (counts per $\mathrm{cm}^{2}$ of CCD area s ${ }^{-1}$ ) & $2 \mathrm{E} 4-1 \mathrm{E} 9$ \\
JIRAM HgCdTe focal plane array infrared imager & $>5 \mathrm{MeV}$ & $4 \mathrm{E} 5-1 \mathrm{E} 7$ (penetrating events s ${ }^{-1}$ ) & $1.2 \mathrm{E} 6-3 \mathrm{EE7}$ \\
ASC CCD imager (onboard particle counter) & $>10 \mathrm{MeV}$ & $600-10,000$ (reported CCD impacts per image) & $3 \mathrm{E} 5-5 \mathrm{E} 6$ \\
\hline
\end{tabular}

${ }^{a}$ Lower energies are expected to make a relatively small contribution to the count rate in hard spectral environments. Measurement ranges apply for nominal $1 \mathrm{~s}$ JIRAM imager exposures and $125 \mathrm{~ms}$ single field ASC imaging. Quoted ASC and JIRAM ranges are very conservative; quantitative measurements outside these ranges may be possible following additional calibrations.

independent of this issue, environment model uncertainties are present within our baseline metrics used to convert count rates to external integral fluxes.

Simultaneous observations gathered by RM instruments provide comparative spectral information due to differing spectral sensitivities brought about by significant differences in instrument shielding (Figure 2). $\mathrm{RM}$ instruments detect penetrating electrons from rather broad energy channels ( $>5 \mathrm{MeV}$ and $>10 \mathrm{MeV}$ ) and a distribution of penetration efficiency energy "bins" exists within each channel. The fractional contribution to the total count rate by a given energy bin will vary as the spectral hardness of the ambient particle population changes. Simultaneous measurements provide the opportunity to study these higher external energies by comparing the responses of the different instruments at the same sampling locations. ASC count rates are significantly influenced by the $>20 \mathrm{MeV}$ electron population, whereas the SRU exhibits a more gradual increase in sensitivity to higher energies due to its very different shielding configuration. JIRAM is relatively more sensitive at energies $<10 \mathrm{MeV}$ and provides added information about this part of the external spectra.

\section{Observations}

The SRU, ASC, and JIRAM imagers made coordinated measurements of the Jovian radiation environment during Juno's first science pass over the poles and through the inner magnetosphere on 27 August 2016 (Figure 3a). ASC particle count data (an onboard count of the transient radiation signatures detected within each image field) were collected with $4 \mathrm{~Hz}$ sampling throughout the passage. Six SRU image samples were collected at targeted opportunities within the inner magnetosphere and polar regions (Figure 3b). The significantly lower SRU sampling rate is due to constraints levied by its primary engineering function (to support spacecraft attitude determination). Fifty-one JIRAM images were collected during the observation period, including six coincident with SRU image collection.

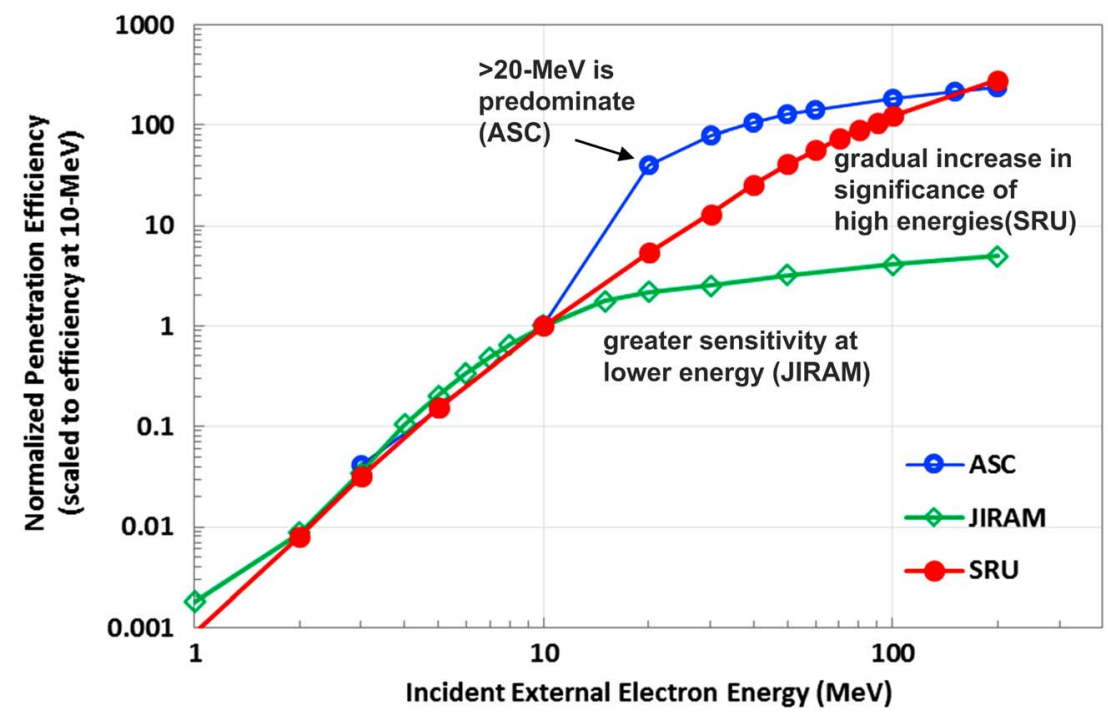

Figure 2. Comparison of spectral sensitivities of the ASC, SRU, and JIRAM. Probabilities for omnidirectional high-energy electrons to penetrate instrument shielding and create image noise events ("penetration efficiencies") have been normalized to the value at $10 \mathrm{MeV}$ for each instrument. 


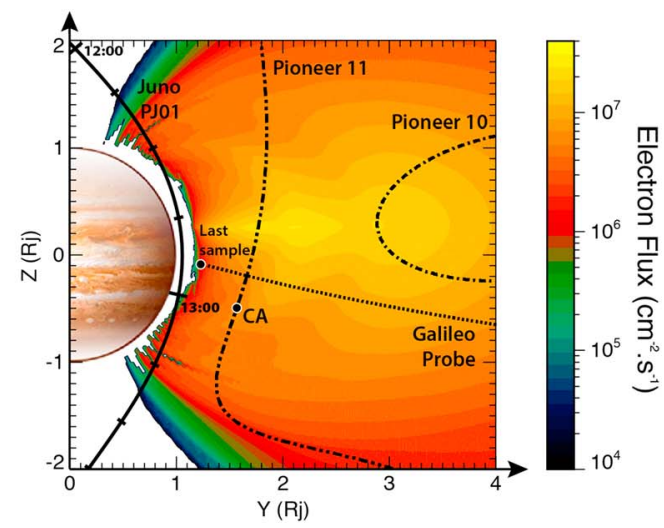

(a)

VLA Observation at $1400 \mathrm{MHz}$ (May 1997) at $\mathrm{CML}($ deg. $)=183.29 \quad$ [ WLong (deg.) $=93.29$ ]

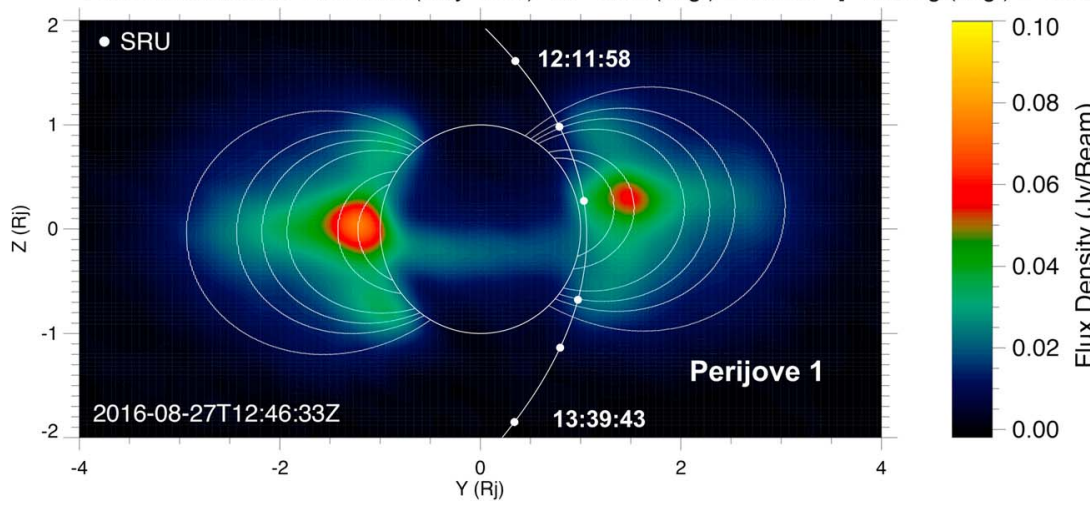

(b)

VLA Observation at $1400 \mathrm{MHz}$ (May 1997) at $\mathrm{CML}$ (deg.) = 94.46 [ WLong (deg.) = 4.46 ]

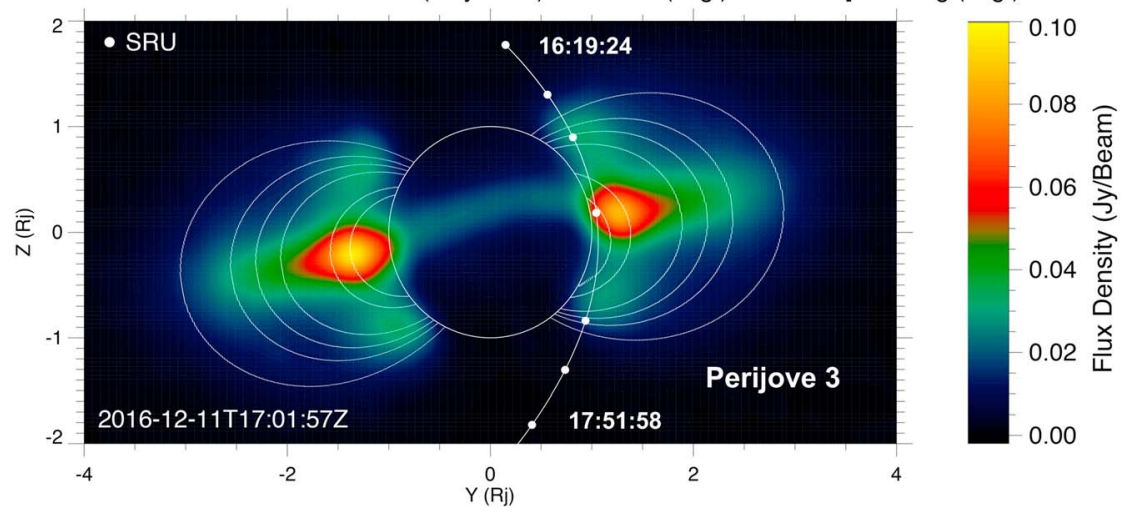

(c)

Figure 3. (a) Juno's 27 August 2016 "Perijove 1" trajectory. Times are in UTC with tick marks at 15 min intervals. Juno's range at closest approach (12:50:44 UTC) was $1.06 R ; ; 147 \mathrm{~km}$ from the cloud tops. Colored contours are $>10 \mathrm{MeV}$ electron fluxes from Santos-Costa and Bolton [2008]. Perijove 1 was a first look at territory not sampled by prior in situ instruments (trajectories shown). Markers indicate the range of the Pioneer 11 spacecraft at its closest approach (CA), and the last sample collected by the Galileo Probe. ( $b$ and c) SRU image collection locations (white dots) along Juno's Perijove 1 and Perijove 3 science passes. Flux density of synchrotron emission is shown $\left(\sim 21 \mathrm{~cm}\right.$, averaged over $20^{\circ}$ of longitude) for context. Synchrotron map projections [after Santos-Costa et al., 2014] are for the central meridian longitude corresponding to the System III West longitude of the spacecraft near the time of the magnetic equator crossing of each flyby (crossing times shown in lower left corners of maps). Magnetic field lines highlighting peak emissions are plotted for $L=1.3,1.5,2$, 2.25, 2.5, 3 and were calculated using the VIP4 magnetic field model [Connerney et al., 1998]. SRU image collection was targeted to occur as Juno grazed the inner edges of the high-latitude lobes of the synchrotron region. Positive $X$ axes point toward the observer throughout.

Perijove 3 did not include JIRAM science observations due to a spacecraft engineering issue related to onboard JRAM data management. SRU and ASC data were collected. Figure $3 \mathrm{c}$ shows Perijove 3 SRU imaging locations within the period of 16:15 to 18:00 UTC on 11 December 2016. In addition to $4 \mathrm{~Hz}$ ASC particle count sampling, ASC images collected at the times of SRU imaging were retrieved during Perijove 3 for 



Figure 4. Omnidirectional fluxes of $>5 \mathrm{MeV}$ and $>10 \mathrm{MeV}$ electrons derived from penetrating particle count rates observed by the SRU, ASC, and JIRAM imagers along Juno's (a) Perijove 1 and (b) Perijove 3 trajectories. SRU (red) and JIRAM (green) measurements with count rates lower than the calibrated measurement range, or zero, are marked as crosses. ASC data have been corrected for electronic shutter time and filtered to remove samples affected by straylight. ASC data points at $\sim 10^{4}$ are due to a small star field dependent bias caused by dim stars reported amongst the count of penetrators (established with data collected during Juno's Cruise period outside of the Jovian magnetosphere). Grey bars show regions of $L$ shell (calculated using the VIP4 magnetic field model [Connerney et al., 1998] and the magnetodisc mode of Connerney et al. [1981]) associated with the orbits of several Jovian moons; blue bars mark $L$ shells associated with the main Jovian ring; closest approach is marked "CA." Omnidirectional $>10 \mathrm{MeV}$ electron flux predictions using Santos-Costa and Bolton [2008] (grey dashed lines) and Garrett et al. [2005] (pink dashed lines) with VIP4 are shown for comparison.

SRU observation times up to and including Juno's passage through the northern high-latitude lobe of the synchrotron region. Ground processing of the ASC images confirmed the count rates reported by the ASC on board particle counting algorithms at the same sampling times.

\subsection{Perijove 1 Results}

Figure 4a shows inferred omnidirectional $>5 \mathrm{MeV}$ and $>10 \mathrm{MeV}$ electron fluxes as a function of time derived from JIRAM, ASC, and SRU measurements collected during Perijove 1. Fluxes inferred from the three instruments are remarkably consistent. Rapid changes in flux are observed at high-latitude locations where Juno transits quickly between large $L$ shells and the trapped radiation belts (e.g., 12:13). Fluxes decrease near the orbits of lo and Amalthea and increase radially inward of each satellite's orbital radius. Similar behavior is seen a bit more subtly near the orbit of Thebe. Such microsignatures are expected due to satellite absorption and are predicted by the model of Santos-Costa and Bolton [2008], for instance. Similar dips and peaks were observed in the Pioneer electron flux data [Fillius, 1976] and Galileo probe data [Fischer et al., 1996], attributed to satellite absorption (sweeping) effects [Fillius, 1976]. ASC detected fluxes above its background noise level until 12:35 UTC when Juno reached a range of $1.18 R_{J}$ (dipole magnetic latitude $37.6^{\circ}$ ). Detection of above background count rates resumed outbound from closest approach (CA) at 13:00 UTC (1.1 $R_{\mathrm{J}}$, dipole magnetic latitude $-21.2^{\circ}$ ). 
In Figure 4 we compare RM electron fluxes to omnidirectional $>10 \mathrm{MeV}$ electron fluxes predicted by the physical model of Santos-Costa and Bolton [2008] (grey curves) and the empirical model of Garrett et al. [2005] (pink curves). Differences are observed. The measurements were generally found to be lower than predicted fluxes within the synchrotron region. The discrepancies are largest for spacecraft locations $<1.3$ $R$, corresponding approximately to calculated $L$ shells inside the orbit of Amalthea.

An intense radiation peak was observed in the ASC particle counter data at 13:35:45 UTC. It was accompanied by detection of many bright objects (as yet unidentified) in the field of view of all four ASC camera heads, though not precisely at the same instant; the camera heads scan two distinct directions in the sky. This event occurred as the spacecraft was transiting the southern polar region outbound from Perijove $1\left(1.8 R_{\jmath},-71.8^{\circ}\right.$ dipole magnetic latitude) and coincides with an intense, asymmetric bidirectional electron beam identified by Juno's Jupiter Energetic Particle Detector Instrument (JEDI) [Mauk et al., 2014] at the same time [Mauk et al., 2017]. JEDI investigators noted that the beam occurred near the statistical main auroral oval and appeared to be quite spectrally hard. Neither SRU nor JIRAM images were collected at this particular location; however, a JIRAM image collected immediately following at 13:36:07 UTC contained penetrating particle signatures at a relatively low level, below the calibrated measurement range.

\subsection{Perijove 3 Results}

Figure $4 \mathrm{~b}$ shows derived $>10 \mathrm{MeV}$ electron fluxes as a function of time from ASC particle counter measurements and SRU images collected during Perijove 3. Times during which the spacecraft traversed $L$ shells associated with the Jovian satellites and ring are indicated as in Figure 4a, along with omnidirectional $>10 \mathrm{MeV}$ electron flux model predictions. Similar dips in flux were observed in the vicinities of lo and Amalthea, followed by small peaks inside their respective orbits. As observed with Perijove 1, RM measurements during Perijove 3 indicated lower than anticipated electron fluxes in the innermost radiation belts. In contrast to the model predictions, ASC particle counts dropped off to the background noise level at $16: 53$ at a range of $1.12 R$, and no radiation above the noise level was detected by the ASC in the equatorial region near closest approach (CA at 17:03:40; $1.06 R_{\jmath}, 5.7^{\circ}$ latitude, 5.5 $5^{\circ}$ System III (SIII) west longitude). This is consistent with the energetic electron flux measurements of the Galileo probe energetic particle instrument [Fischer et al., 1996] which showed a disappearance of particles inside $\sim 1.25 R$, at a similar SIII west longitude (4.94 $\mathrm{W}$ longitude, $6.53^{\circ}$ latitude at time of probe entry according to Bell [1996]). The SRU image collected at 17:02:11.981 ( $1.06 R_{J}, 9.5^{\circ}$ latitude, $4.6^{\circ} \mathrm{SIII}$ west longitude) confirmed the absence of detectable high-energy electron flux in this region. The ASC resumed particle detection outbound from periapsis at 17:15, at ranges $>1.12 R$. The absence of most ASC data points between 16:53 and 17:15 in Figure $4 \mathrm{~b}$ is due to the filtering out of samples contaminated by straylight.

The ASC again reported intense bursts of penetrating particle counts as Juno transited Jupiter's polar regions during the Perijove 3 flyby. Events were observed in the north at 15:40 and from 15:54 to 16:17, as well as at 17:38 soon after exiting the radiation belts in the south.

\section{Discussion and Summary}

The differences seen between the model predictions and RM's inferred $>10$ MeV electron flux measurements suggest that RM measurements will be very beneficial in improving our understanding of electron energy spectra and pitch angle distributions close to the planet. Both models predicted higher integral fluxes than observed, by up to a factor of $\sim 5$ at high latitudes close to the planet on $L$ shells inside lo's orbit. Flux predictions reach over an order of magnitude higher than the measurements inside the orbit of Amalthea where Juno's radial distance decreases to $<1.3 R$ J. This could indicate that pitch angle distributions are different than predicted (affecting the number of particles bouncing along the field lines Juno crosses at high latitudes), or that loss mechanisms with energy and pitch angle dependence are underestimated (e.g., particle precipitations into loss cones or losses from the emptying of drift shells).

Measured departures of the magnetic field from VIP4 model predictions (discussed in Bolton et al. [2017]) may also contribute to the differences, as VIP4 field predictions were used to derive the modeled fluxes. As noted by Bolton et al. [2017] the magnetic field is not well characterized close to the planet by existing field models, given limited prior observations near Jupiter's surface. 
Empirical models of the radiation belts such as Garrett et al. [2005] depend on fitting parameters which are constrained by in situ data and field model assumptions. Field model inaccuracies and the absence of constraining in situ data can produce incorrect fitting parameters for unexplored regions. Physics-based models such as Santos-Costa and Bolton [2008] face similar challenges as they also depend on magnetic field models, initial conditions constrained by in situ data, and assumptions about modes of transport and diffusive and nonstochastic loss mechanisms. Without prior in situ data or a realistic field model to constrain radiation belt models close to the planet, the discrepancies seen between predictions and measurements are possible.

RM count rate measurement accuracy is good to within 22\% [Becker et al., 2017] and the measurements of the three instruments are very consistent; therefore, the differences between predicted and measured counts suggest to us that the intensity or spectral distribution of electrons $>5 \mathrm{MeV}$ is different than expected close to the planet. Differential intensities and energy spectra above $10 \mathrm{MeV}$ can be estimated where simultaneous observations by the RM instruments are available, given their different sensitivities to high-energy particles. Future work will utilize the integral $>5 \mathrm{MeV}$ and $>10 \mathrm{MeV}$ intensities shown here, as well as our knowledge of the differing sensitivities of the instruments to energies above $10 \mathrm{MeV}$ (Figure 2). The SRU and ASC show differing responses during some of the coordinated observations. This could provide additional spectral constraints for these locations. Electron flux data from the JEDI investigation ( 20-1000 keV) will further guide the analysis, and Juno's ongoing longitude mapping of the inner magnetosphere will be very important to our understanding of the data. The Magnetic Field Investigation, JEDI, and Microwave Radiometer investigation will provide key data sets.

We have presented in situ measurements of $\mathrm{MeV}$ electrons derived using penetrating particle signatures in images from Juno's heavily shielded instruments. Coordinated observations provide spectral information given differing instrument shielding and sensitivity. Signatures suggestive of satellite absorption were observed. Juno is the first spacecraft to explore the inner edge of the high-latitude lobes in situ, and measured MeV fluxes were lower than predicted. RM measurements show great promise in aiding our understanding of Jupiter's radiation belts, particularly within the inner edges of Jupiter's radiation belts.

Enhanced particle counts were observed while crossing Jupiter's polar regions. In future orbits, a greater number of SRU images will be collected near expected crossings of the northern and southern auroral ovals (coordinated with JIRAM and ASC imaging when compatible) to corroborate inferred flux levels detected by the onboard ASC particle counter and aid the further study of the energy spectra of these events.

\section{Acknowledgments}

This work was sponsored by the Jet Propulsion Laboratory, California Institute of Technology, under a contract with the National Aeronautics and Space Administration. The JIRAM Project is funded by the Italian Space Agency. The data currently reside at the Jet Propulsion Laboratory. Contact Heid N. Becker (Heidi.N.Becker@jpl.nasa.gov) for access to data.

\section{References}

Adriani, A., et al. (2014), JIRAM, the Jovian Infrared Auroral Mapper, Space Sci. Rev., doi:10.1007/s11214-014-0094-y. Allison, J., et al. (2006), Geant4 developments and applications, IEEE Trans. Nucl. Sci., 53(1), 270-278, doi:10.1109/TNS.2006.869826. Becker, H. N., et al. (2017), The Juno Radiation Monitoring (RM) Investigation, Space Sci. Rev., doi:10.1007/s11214-017-0345-9.

Bell, J. L. (1996), Galileo navigation team file release: Final updated ephemeris file for probe reconstruction JPL doc. [960730-OD126], Jet Propul. Lab., Pasadena, Calif. [Available at http://naif.jpl.nasa.gov/pub/naif/GLL/kernels/spk/s960730a.bsp.lbl.]

Bolton, S. J., R. M. Thorne, S. Bourdarie, I. DePater, and B. Mauk (2004), Jupiter's Inner Radiation Belts, in Jupiter, the Planet, Satellites and Magnetosphere, edited by F. Bagenal, T. E. Dowling, and W. B. McKinnon, pp. 671-688, Cambridge Univ. Press, New York.

Bolton, S. J., et al. (2017), Jupiter's interior and deep atmosphere: The initial pole-to-pole passes with the Juno spacecraft, Science, doi:10.1126/science.aal2108.

Carr, T. D., M. D. Desch, and J. K. Alexander (1983), Phenomenology of Magnetospheric Radio Emissions, in Physics of the Jovian Magnetosphere, edited by A. J. Dessler, pp. 226-284, Cambridge Univ. Press, Cambridge, New York.

Connerney, J. E. P., M. H. Acuña, and N. F. Ness (1981), Modeling the Jovian current sheet and inner magnetosphere, J. Geophys. Res., 86, $8370-8384$.

Connerney, J. E. P., M. H. Acuña, N. F. Ness, and T. Satoh (1998), New models of Jupiter's magnetic field constrained by the lo flux tube footprint, J. Geophys. Res., 103(A6), 11929-11939, doi:10.1029/97JA03726.

Connerney, J. E. P., et al. (2017), The Juno Magnetic Field Investigation, Space Sci. Revs., doi:10.1007/s11214-017-0334-z.

Divine, N., and H. B. Garrett (1983), J. Geophys. Res., 88, 6889-6903.

Fillius, W. (1976), The trapped radiation belts of Jupiter, in Jupiter, edited by T. Gehrels, pp. 896-927, Univ. of Arizona Press, Tuscon.

Fischer, H. M., E. Pehlke, G. Wibberenz, L. J. Lanzerotti, and J. D. Mihalov (1996), High-energy charged particles in the innermost Jovian magnetosphere, Science, 272, 856-858.

Garrett, H. B., I. Jun, J. M. Ratliff, R. W. Evans, G. A. Clough, and R. W. McEntire (2003), Galileo Interim Radiation Electron Model, Jet Propul. Lab., Calif. Inst. of Technol., JPL Publ. 03-006, Pasadena, Calif.

Garrett, H. B., S. M. Levin, S. J. Bolton, R. W. Evans, and B. Bhattacharya (2005), A revised model of Jupiter's inner electron belts: Updating the Divine radiation model, Geophys. Res. Lett., 32, L04104, doi:10.1029/2004GL021986.

Garrett, H. B., M. Kokorowski, I. Jun, and R. W. Evans (2012), Galileo Interim Radiation Electron Model: Update-2012, Jet Propul. Lab., Calif. Inst. of Technol., JPL Publ. 12-9, Pasadena, Calif.

Mauk, B. H., et al. (2014), The Jupiter Energetic Particle Detector Instrument (JEDI) investigation for the Juno mission, Space Sci. Rev., doi:10.1007/s11214-013-0025-3. 
Mauk, B. H., et al. (2017), Juno observations of energetic charged particles over Jupiter's polar regions: Analysis of monodirectional and bidirectional electron beams, Geophys. Res. Lett., 44, doi:10.1002/2016GL072286.

Santos-Costa, D., and S. J. Bolton (2008), Discussing the processes constraining the Jovian synchrotron radio emissions features, Planet. Space Sci, 56, 326.

Santos-Costa, D., I. de Pater, R. J. Sault, M. A. Janssen, S. M. Levin, and S. J. Bolton (2014), Multifrequency analysis of the Jovian electron-belt radiation during the Cassini flyby of Jupiter, Astron. Astrophys., 568, A61, doi:10.1051/0004-6361/201423896. 